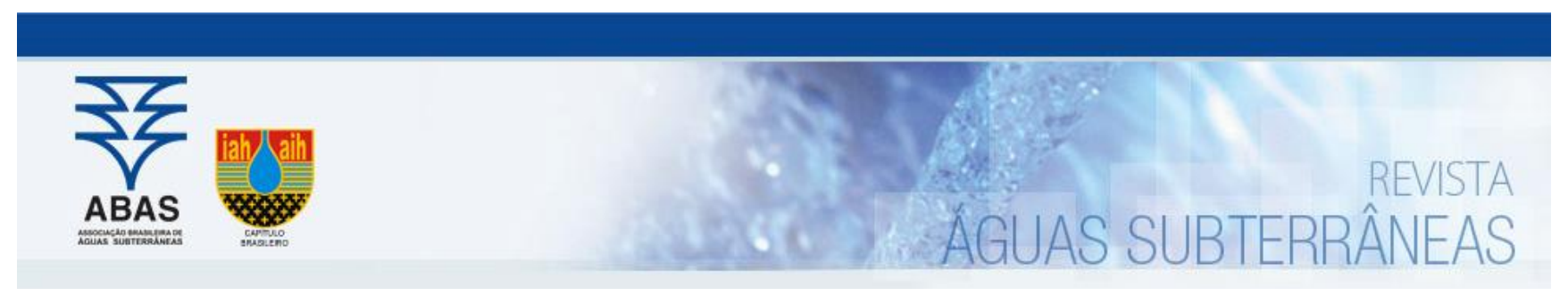

Artigos

\title{
Aplicação de mapas auto-organizáveis e SIG: análise espacial da hidroquímica dos aquíferos em uma média cidade brasileira
}

\section{Application of self-organizing maps and GIS: spatial analysis of hydrochemistry of the aquifers in a Brazilian medium-size city}

\author{
Jonatas Batista Mattos; Kaique Brito Silva2 ${ }^{\bowtie}$ \\ 1 PPGeo. Instituto de Geociências, Universidade Federal da Bahia (UFBA), Salvador, BA \\ 2 Leclig. Instituto de Geociências, Universidade Estadual de Campinas (Unicamp), Campinas, SP
}

$\triangle$ jon.geociencia@gmail.com,_kbritofb96@hotmail.com

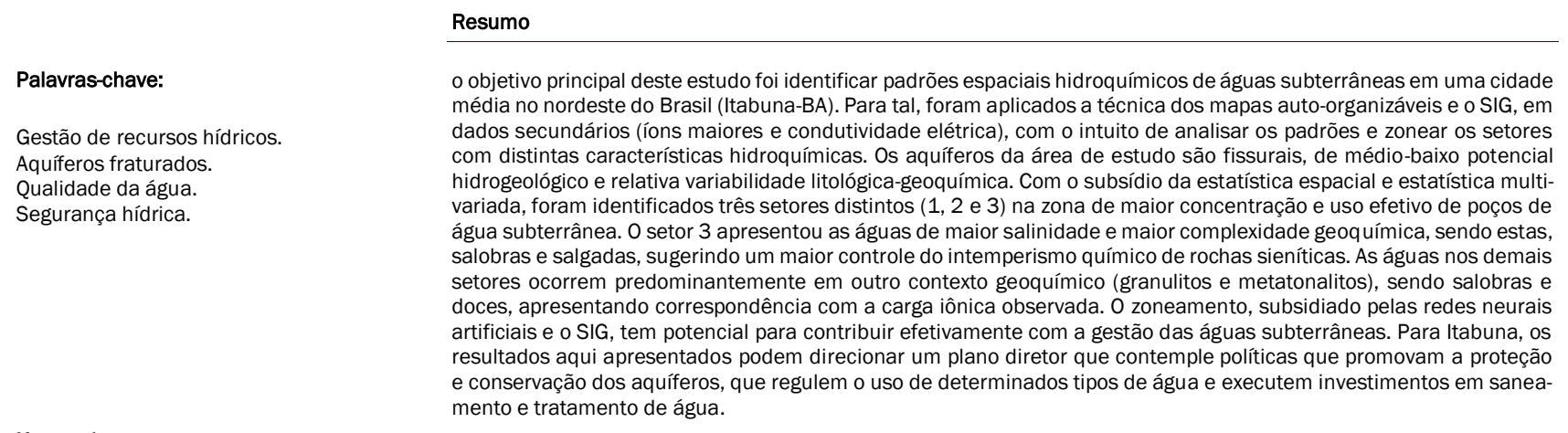

\section{Keywords:}

Water management.

Fractured aquifers.

Water quality.

Water security.

Revisado por pares.

Recebido em: 04/07/2018.

Aprovado em: 02/05/2019.

\begin{abstract}
The objective of this study was to identify hydrochemical spatial patterns of groundwater; n northeastern Brazil (ItabunaBA). For this, the self-organizing maps and the GIS were applied in secondary data (major ions and electrical conductivity), with the purpose of analyzing the patterns and zoning the sectors with different hydrochemical characteristics. The aquifers of the study area are fractured, medium-low hydrogeological potential and relative lithological-geochemical variability. With the subsidy of spatial statistics and multivariate statistics, three distinct sectors $(1,2$ and 3$)$ were identified in the zone of greatest concentration and effective use of groundwater wells. Sector 3 presented water with higher salinity and greater geochemical complexity, being these brackish and salted, suggesting a greater control of the chemical weathering of sienitic rocks. The waters in the other sectors occur predominantly in another geochemical context (granulites and metatonalites), being brackish and freshwater, presenting correspondence with the observed ionic load. Zoning, subsidized by artificial neural networks and GIS, has the potential to contribute effectively to groundwater management. For Itabuna, the findings presented here may subsidize to a master plan that contemplates policies that promote the protection and conservation of aquifers that regulate the use of certain types of water and carry out investments in sanitation and water treatment.
\end{abstract}

DOI: http:/dx.doi.org/10.14295/ras.v33i2.29176

\section{INTRODUÇÃO}

O abastecimento de significativa parcela da população mundial depende diretamente das águas subterrâneas (MARGAT \& VAN DER GUN, 2013; GLEESON et al., 2014), e entender os processos que controlam estas águas é tão essencial quanto compreender os padrões ambientais e antrópicos que se revelam em uma determinada unidade espacial (BARROS et al., 2015; GUDE, 2018). Para tal, a gestão destas águas deve ser pensada a partir de abordagens inteligentes e objetivas que identifiquem os padrões, atenuem impactos, estabeleçam diretrizes, e por consequência, promovam a segurança hídrica.

Devido à rápida inovação da tecnologia computacional, técnicas 
e algoritmos para tratamento e representação de dados complexos podem ser facilmente gerenciados. O Sistema de Informação Geográfica (SIG) e as redes neurais artificiais, particularmente, os mapas auto-organizáveis (SOM), são exemplos de ferramentas que podem fornecer o subsidio necessário para resolução de problemas com as águas subterrâneas (MACHIWAL \& JHA, 2015; BELKHIRI et al., 2018). Complexos processos geoquímicos naturais, somados a processos tipicamente antrópicos (urbanização, indústria e agricultura), estabelecem as características químicas das águas subterrâneas, que precisam ser monitoradas para interpretação e definição de usos possíveis (MELO et al., 2018; HASSANE et al., 2016). Geralmente, o monitoramento hidroquímico destas águas, envolve um grande número de amostras, dimensões e variáveis, que precisam ser otimizadas para análises, e o SOM associado ao SIG pode detectar relações não-lineares e espaciais entre os dados.

Como os SOM fornecem elementos de estatística multivariada e realidade multidimensional (FRIEDEL et al., 2012; SEGEV \& KANTOLA, 2012), esta metodologia, além de orientar a interpretação dos processos hidrogeoquímicos, pode refinar análises espaciais e mapeamentos executados pelo SIG. A aplicação combinada destas técnicas pode ser uma forma eficaz de conhecer as zonas prioritárias para gestão, dentro de uma abordagem que considera questões de abastecimento e qualidade da água subterrânea com elementos sociais e ambientais.
No período de 2015-2016, a cidade de Itabuna-BA, vivenciou a pior crise hídrica de sua história (MATTOS et al., 2019). A falta de água durou meses, e a pouca água disponível era de péssima qualidade. Por ser uma região que historicamente chove muito e os regimes fluviais são perenes, a água superficial sempre foi a matriz do abastecimento local, entretanto, desde a crise hídrica, a procura e o uso de água subterrânea aumentou significativamente. Neste cenário, a busca pelo entendimento dos processos geológicos e ambientais que controlam estas águas é fundamental para delimitar as potencialidades e limitações de uso. Assim, para fornecer ferramentas que subsidie a gestão dos recursos hídricos em um território, o objetivo principal deste estudo foi identificar padrões espaciais hidroquímicos das águas subterrâneas de aquíferos fissurais em Itabuna a partir da aplicação e associação de estatística espacial (SIG) e estatística multivariada (SOM).

\section{2. ÁREA DE ESTUDO}

O município de Itabuna, localiza-se no sul do estado da Bahia (microrregião econômica Ilhéus-Itabuna), entre as coordenadas 1441', 15²'S e 39¹3', 39³0'W (Figura 1). É uma média cidade que, de acordo com o IBGE (2017), apresenta uma população estimada em 221.046 habitantes, estando entre os trinta mais populosos municípios da região Nordeste.

Figura 1 - Mapa de localização

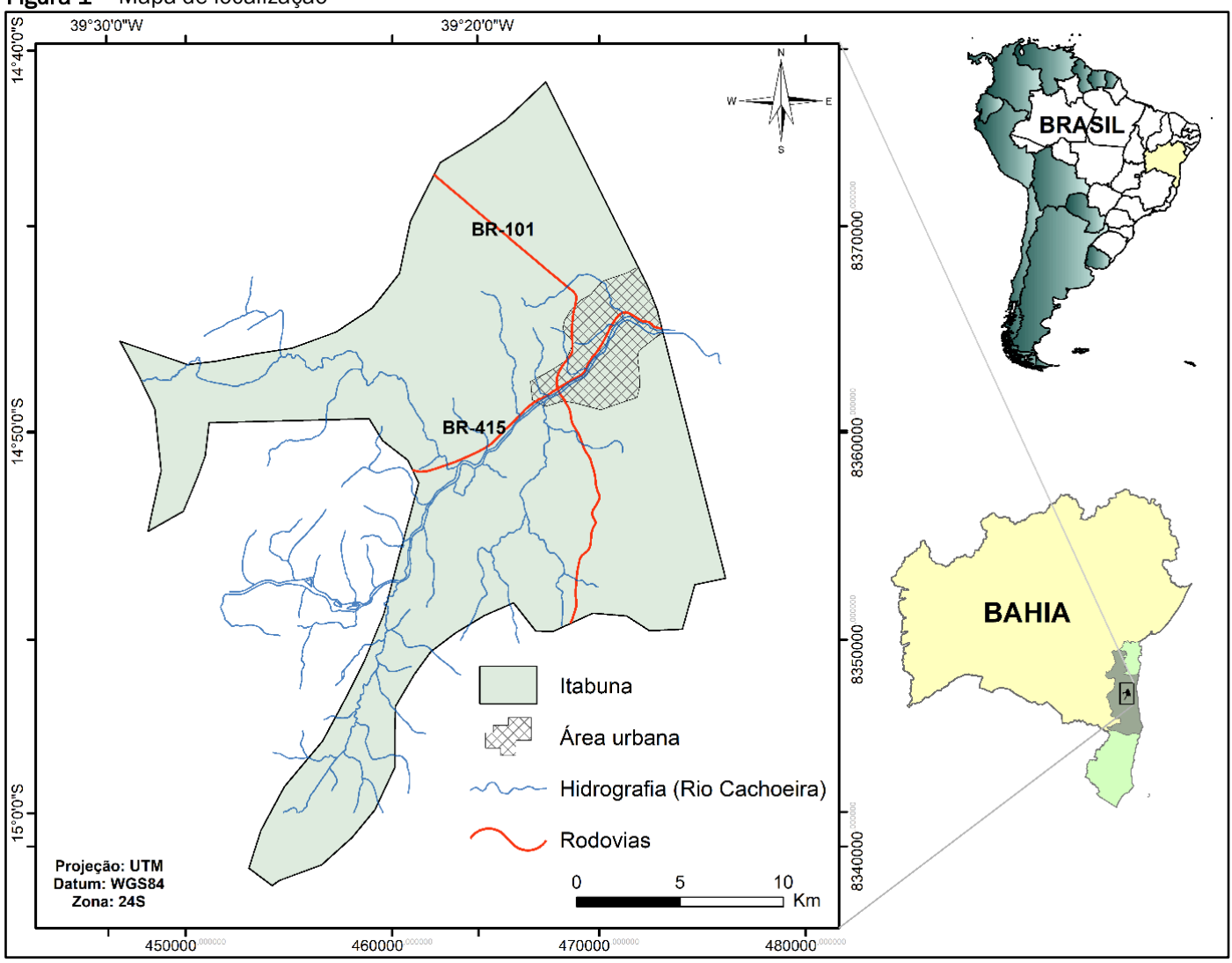


A média histórica de chuvas no município gira em torno de 1.500 $\mathrm{mm}$, segundo registros do INMET. De acordo com a classificação internacional de Koppen (1948), o tipo climático observado na região é $A f$, classificado como tropical úmido de floresta sem estação seca definida. Segundo Alvares et al. (2013), esse clima caracteriza-se também por temperatura média de $24^{\circ} \mathrm{C}$, e precipitação anual acima de $2.000 \mathrm{~mm}$. Ainda segundo o autor, os valores de chuva indicam que nessa região ocorrem algumas zonas (isolinhas) com níveis pluviométricos similares ao clima amazônico.

O contexto geológico no qual está inserido a área de estudo cor- responde a faixa leste do Cráton do São Francisco, estabilizada desde a Era Proterozóica. A litologia é composta em grande parte por granulitos, metatonalitos e intrusões de rochas alcalinas-sieníticas (TERAMOTO et al., 2018). Já as águas subterrâneas, de acordo com Maia et al. (2009), ocorrem em domínios de rochas do embasamento cristalino, o que determina aquíferos dos tipos fissurais, de baixo a médio potencial hidrogeológico. A Figura 2 mostra uma síntese litológica-hidrogeológica com os pontos-poços, que ilustra todo o contexto que determina a hidroquímica na área de estudo.

Figura 2 - Mapa de síntese litológica-hidrogeológica

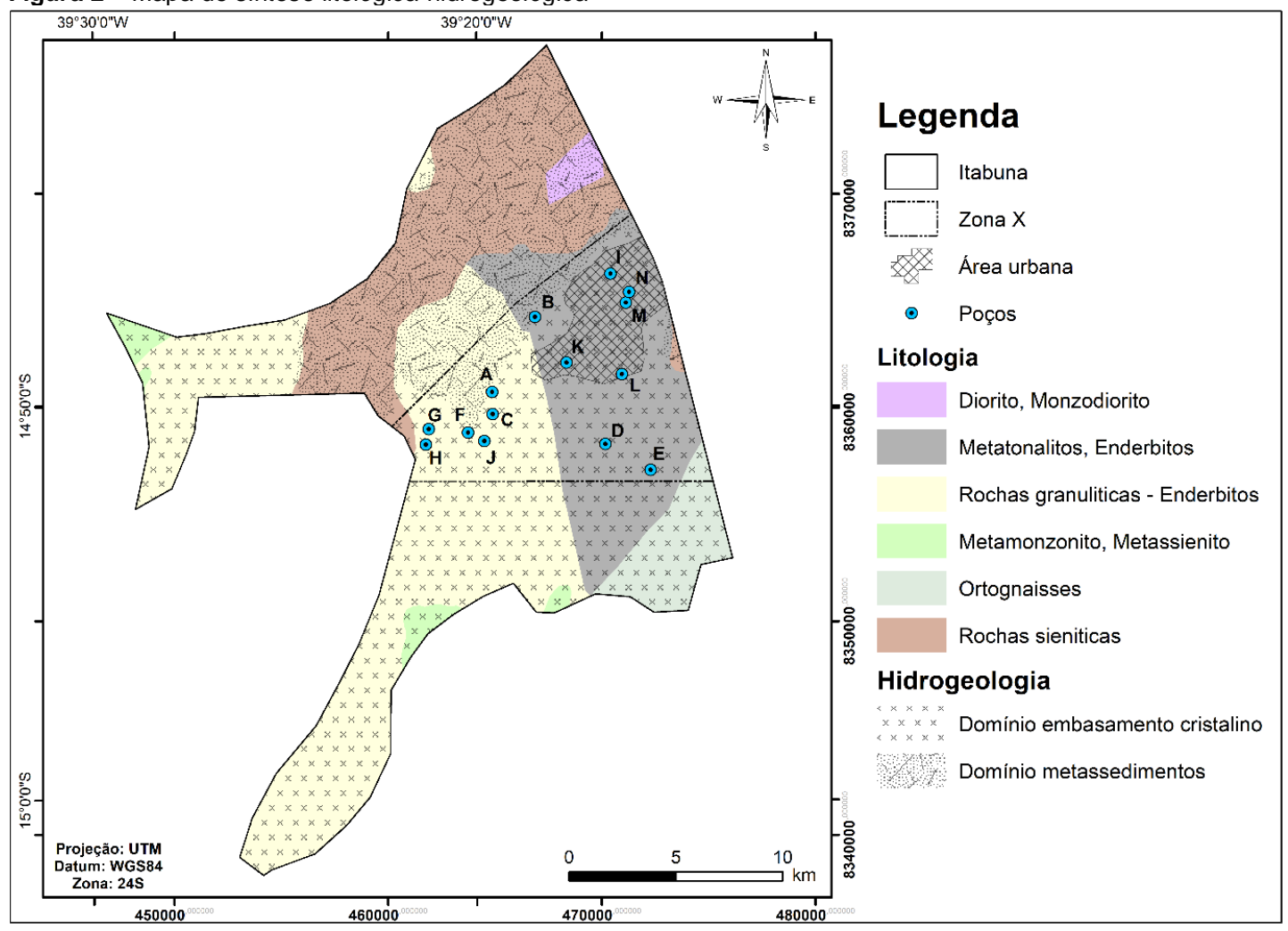

\section{MATERIAIS E METODOS}

\subsection{Procedimentos e dados}

Para este estudo, os dados usados foram secundários, a partir de um estudo conduzido por Teramoto et al. (2018), que interpretou as características hidroquímicas nos aquíferos fissurais da região de Itabuna. De acordo com os autores, foram coletadas amostras de águas de 29 poços para análises laboratoriais de íons maiores e isótopos. Alguns parâmetros como temperatura, pH e condutividade elétrica foram obtidos ainda em campo. Como o objetivo foi identificar padrões espaciais hidroquímicos especificamente no território de Itabuna, foi feita uma síntese dos 29 pontos de amostragem. Alguns poços estão espacialmente muito próximos, implicando negativamente nas técnicas espaciais empregadas. Além da relação espacial, estes poços, apresentaram similaridades nos valores hidroquímicos, o que forneceu a sustentação para sintetiza-los em menos pontos, a partir da média dos valores observados. Alguns outros poços não foram usados neste estudo, pois, estão alocados no território de municípios vizinhos.

Por fim, a quantidade de pontos para este estudo será 14. Pela irregular distribuição espacial dos pontos, foi delimitada uma zona (Zona X - vide Figura 2), para otimizar o processo de análise espacial. Esta delimitação foi determinada com base no setor de maior concentração dos poços e uso efetivo das águas subterrâneas.

\subsection{SIG e Mapas auto-organizáveis}

Com o uso do SIG, foi executado uma análise espacial para os dados de condutividade elétrica, com o objetivo de subsidiar o processo de setorização da unidade espacial analisada. Cabe 
destacar que, a condutividade elétrica é um parâmetro que está correlacionado com a presença de sais dissolvidos, logo, é representativo para a análise da carga iônica. Para realizar a análise espacial foi usado o Software ArcGis $10.3^{\circledR}$, no qual foi aplicado o método de interpolação pelo inverso do quadrado da distância (Inverse Distance Weighted - IDW). O histograma foi moldado a partir do cálculo de desvio padrão, que ajudou a refinar os limites das classes geradas no mapa.

Para aperfeiçoar o processo de definição dos setores hidroquímicos, foi usado o método das redes neurais artificiais pela técnica dos mapas auto-organizáveis (SOM) de Kohonen (1982), que é estruturado por uma arquitetura específica e por um treinamento não supervisionado. 0 algoritmo base do SOM usa o sistema de aprendizagem por competição, o qual avalia os neurônios (nós) de saída da rede, determinando uma competição entre estes, sendo o neurônio vencedor aquele que apresentar uma maior carga sináptica (KOHONEN, 2013). Os algoritmos calculam as distâncias euclidianas entre cada vetor de dados e todos os vetores de peso de acordo com o modelo de melhor combinação. Este tipo de rede neural pode projetar dados complexos e multidimensionais em uma matriz de duas camadas, regularmente organizada em proporção ao grau de similaridade, sendo capaz de agrupar, predizer e minerar dados de grande variabili- dade (SEGEV \& KANTOLA, 2012; KOHONEN, 2013).

A estatística por trás do SOM é multivariada, e a população amostral usada contém dados de íons maiores $\left(\mathrm{HCO}_{3}, \mathrm{SO}_{4}, \mathrm{Cl}\right.$, $\mathrm{NO}_{3}, \mathrm{Na}^{+}, \mathrm{K}^{+}, \mathrm{Ca}^{2+}, \mathrm{Mg}^{2+}$ ) e da condutividade elétrica, fornecendo maior sustentação para a delimitação dos setores a partir da hidroquímica. 0 procedimento de treinamento da rede neural e geração dos SOM foram realizados através do software MatLab $^{\circledR}$, versão R2018a (THE MATHWORKS INC., 2018).

\section{RESULTADOS E DISCUSSÃO}

\subsection{Análises espaciais}

A Figura 3 ilustra um mapa que espacializa a condutividade elétrica nas águas subterrâneas de Itabuna, no qual, é possível notar que na zona oeste do município ocorrem águas mais salinizadas. Este padrão já tinha sido observado por De Paula et al. (2009), confirmando que esta zona apresenta uma característica geoquímica bastante distinta. As águas nas demais zonas ocorrem predominantemente em outro contexto geoquímico (granulitos e metatonalitos), apresentando correspondência com a condutividade elétrica observada.

Figura 3 - Mapa de distribuição espacial da condutividade elétrica na Zona X

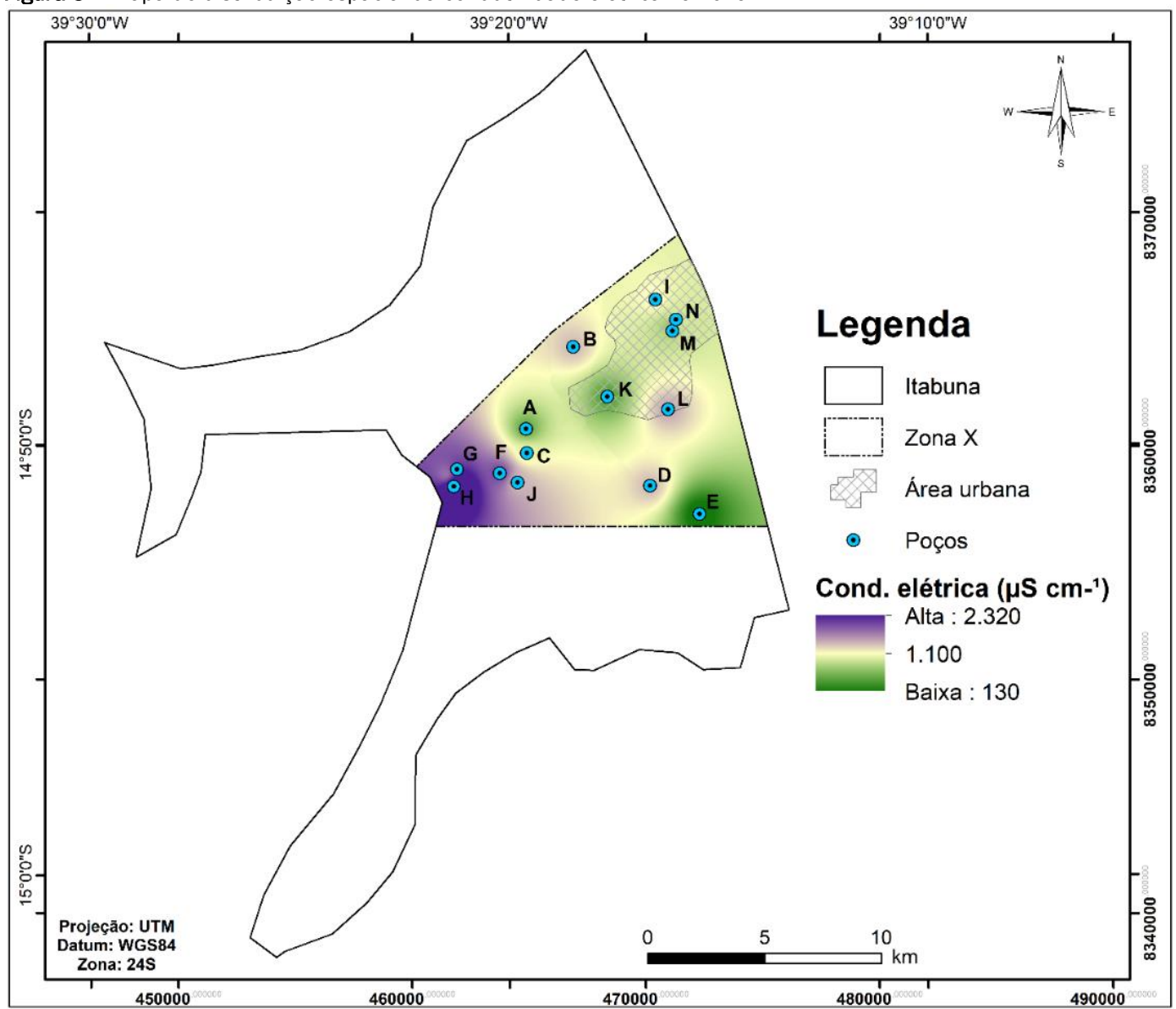


$\mathrm{Na}$ área urbana, ocorrem águas com diferentes teores de salinidade, sugerindo que há diferentes fontes (possivelmente antrópicas) que influenciam na hidroquímica das águas subterrâneas. De forma geral, é corriqueiro encontrar anomalias hidroquímicas em áreas urbanas (GOMES \& CAVALCANTE, 2015; GOMES et al., 2018), sobretudo em um cenário onde as águas residuárias podem ser as razões destas anomalias conforme expõe Hassane et al. (2016). Importante salientar que, para Itabuna, é preciso um monitoramento específico para as águas subterrâneas na área urbana, com análise de maior número de pontos-poços e amostras, além de maior período para identificar as variações espaço temporais e assim identificar um padrão hidroquímico mais consistente.

\subsection{Zoneamento hidroquímico}

Para refinar os padrões espaciais e estabelecer a divisão dos setores no território municipal, foram formulados mapas autoorganizáveis a partir das variações, similaridades e correlações entre os pontos, a condutividade elétrica e os íons maiores (Figura 4). Cada matriz da Figura 4 (a), representa o valor dos vetores de referência dos componentes para 36 possíveis posições, nos quais os vetores foram padronizados para variar de 0 a 1, usando tons de cores quentes. As posições que estão em amarelo são correspondentes aos pontos de maior valor e as que estão em preto para os de menor valor. Cada ânion e cátion apresentam suas cargas sinápticas, revelando o comportamento hidroquímico para cada um dos 14 pontos analisados.
A Figura 4 (b), mostra a distribuição matricial destes pontos, que ilustra um padrão transversal para divisão de três setores. É possível notar com a observação simultânea da figura 4 (a) e 4 (b), que esta distribuição baseada na carga iônica apresenta um padrão similar ao observado no mapa da Figura 3, reforçando a capacidade da condutividade elétrica em representar padrões hidroquímicos. Importante salientar que, nem sempre esta correspondência cartesiana-cartográfica de padrões ocorrerá, entretanto, a estatística multivariada do SOM indicará uma estimativa de delimitação mais precisa, independente do padrão espacial observado por uma análise espacial simples no SIG.

A partir dos dados químicos observados por Teramoto et al. (2018), foram calculados os valores de STD (Sólidos Totais Dissolvidos) e dureza total de $\mathrm{CaCO} 3$ (relação entre sódio, cálcio e magnésio). A Figura 5 mostra a relação entre estes dois parâmetros, indicando que, em Itabuna, as águas subterrâneas de maior teor de sais dissolvidos apresentam uma caraterística de águas salobras e duras. Esta configuração quando analisada pela resolução CONAMA 357/2005 (BRASIL, 2005) para salinidade, reforça o padrão observado pelos SOM. As águas assumem diferentes tipos quanto aos íons maiores (Tabela 1), sendo esta, uma característica que pode ser explicada, pelos distintos ambientes onde são extraídas estas águas, com diferenças observadas na geoquímica das rochas e no uso da terra. Estes padrões ambientais, certamente, determinam a hidroquímica das águas subterrâneas, conforme mostram Qian et al. (2011), Menezes et al. (2014) e Shuler et al. (2017). 
Figura 4 - (a) Mapas auto-organizáveis para os íons maiores e condutividade elétrica; (b) Padrão espacial para os pontos com os setores correspondentes

(a)
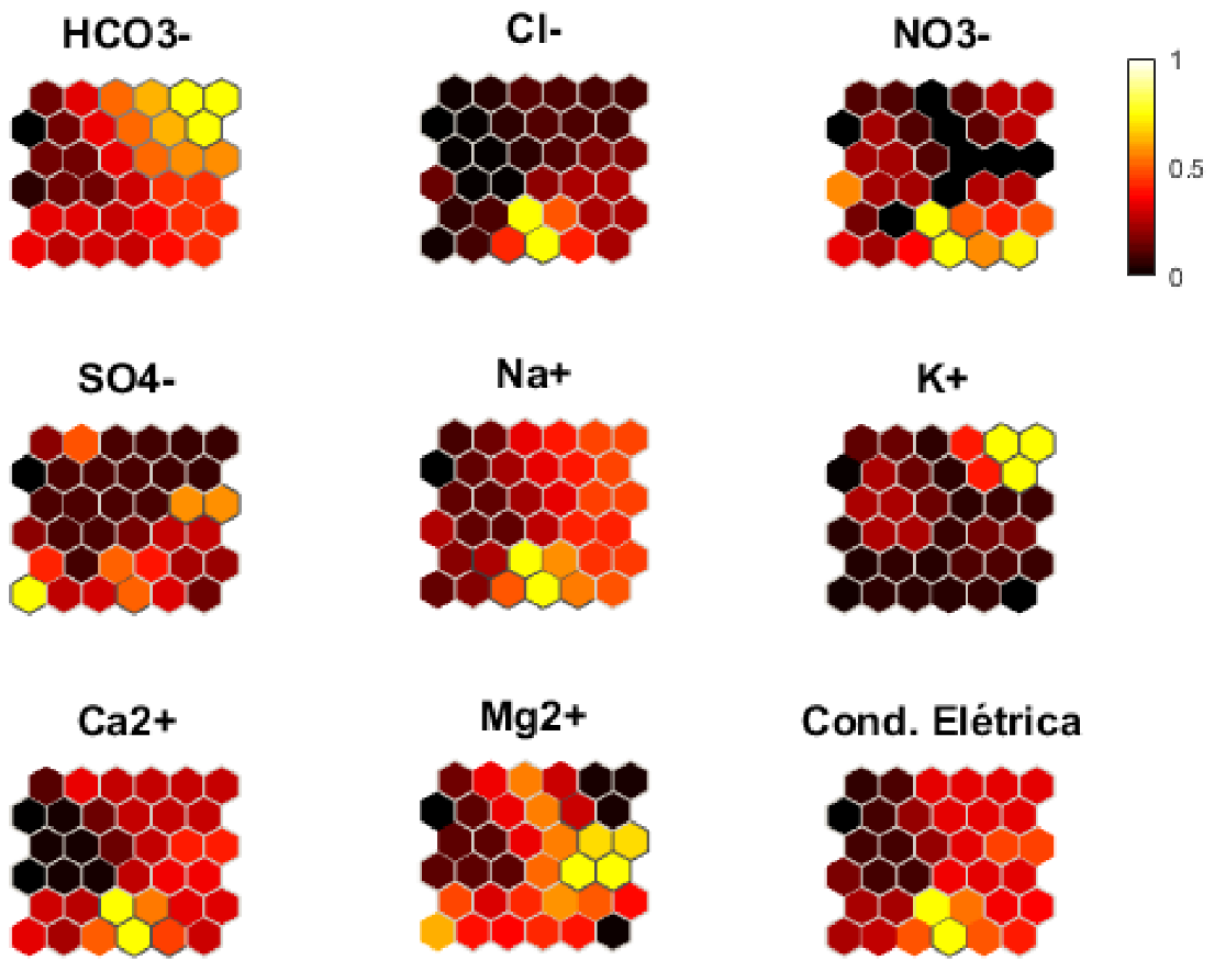

Cond. Elétrica

(b)

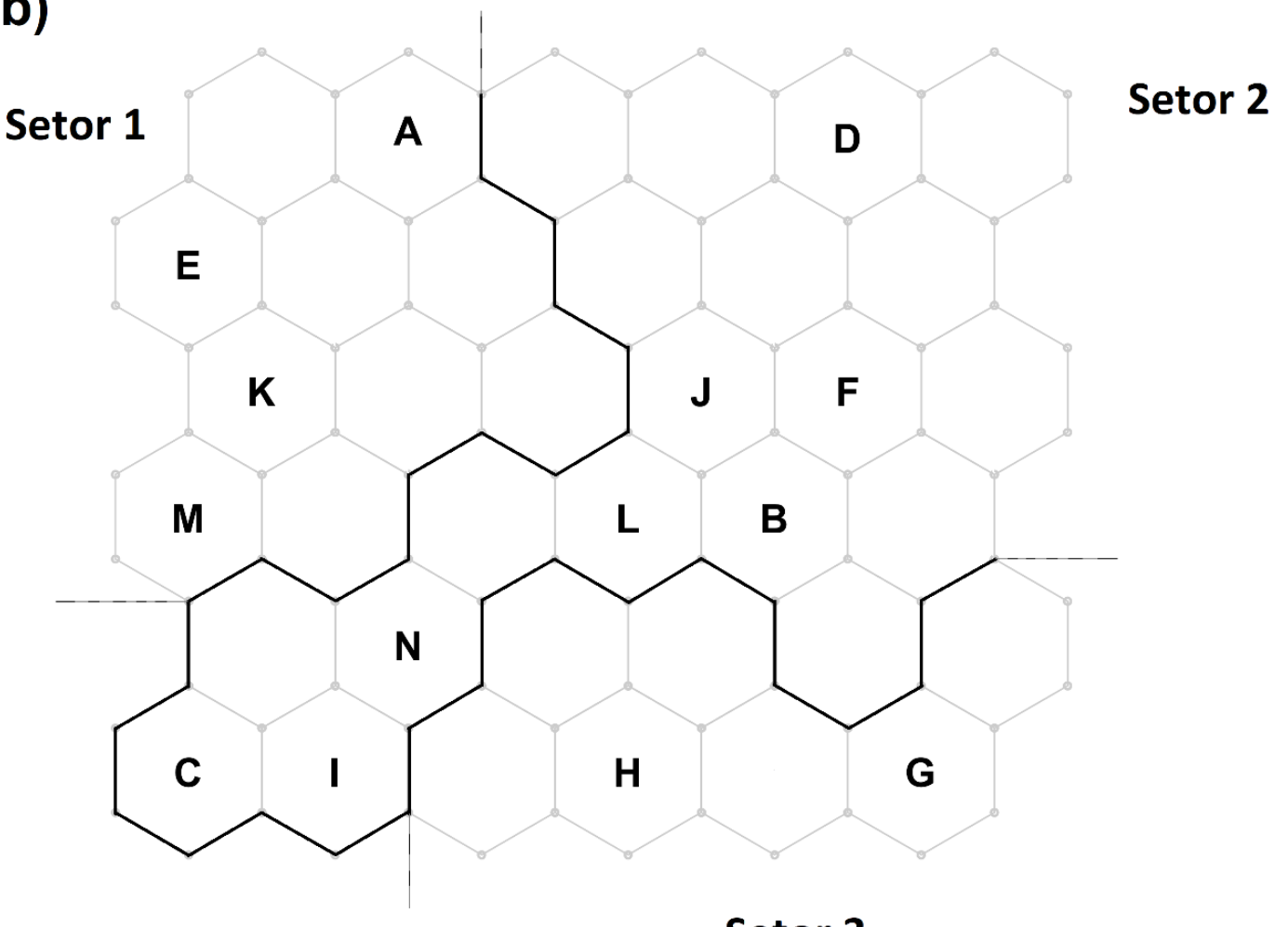

Setor 3 
Figura 5 - Relação entre STD e Dureza Total

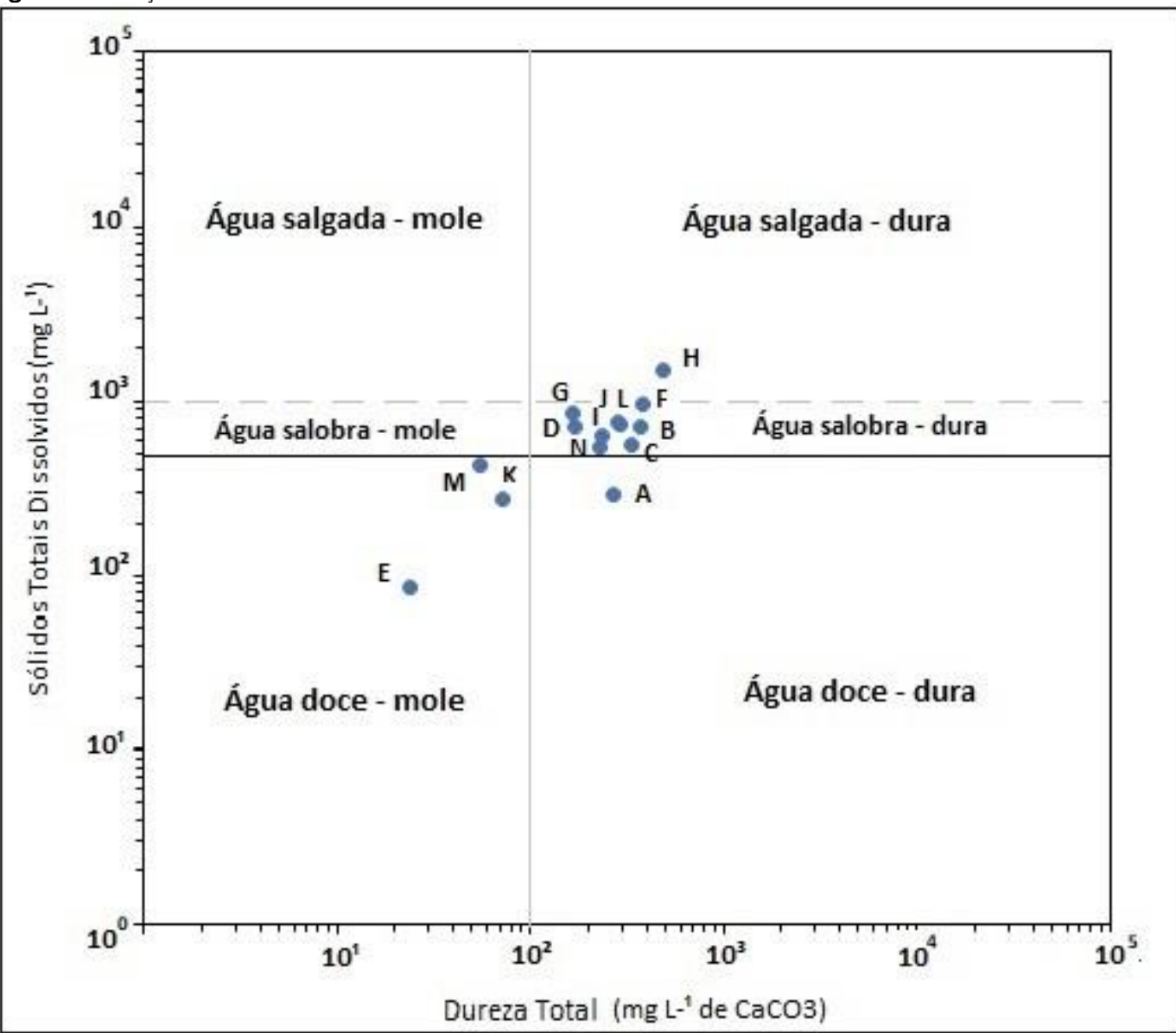

$\underline{\text { Tabela } 1 \text { - Síntese de panorama hidroquímico das águas subterrâneas em Itabuna-BA }}$

\begin{tabular}{|c|c|c|c|c|c|}
\hline \multirow{2}{*}{ Setor } & \multirow{2}{*}{ Pontos } & \multirow{2}{*}{$\begin{array}{l}\text { CE } \\
\left(\mu \mathrm{cm}^{-1}\right)\end{array}$} & \multirow{2}{*}{$\begin{array}{l}\text { Tipos } \\
\text { Piper }\end{array}$} & Salinidade & \multirow[t]{2}{*}{ Dureza T. } \\
\hline & & & & Conama 357/2005 & \\
\hline \multirow{4}{*}{1} & $A$ & 454 & Mista $\mathrm{HCO}_{3}$ & Doce & Dura \\
\hline & $E$ & 129 & Mista $\mathrm{Na}$ & Doce & Mole \\
\hline & $\mathrm{K}$ & 423 & $\mathrm{NaHCO}_{3}$ & Doce & Mole \\
\hline & M & 658 & $\mathrm{NaCl}$ & Doce & Mole \\
\hline \multirow{8}{*}{2} & $\mathrm{~B}$ & 1.111 & $\mathrm{NaHCO}_{3}$ & Salobra & Dura \\
\hline & C & 860 & Mista $\mathrm{HCO}_{3}$ & Salobra & Dura \\
\hline & $\mathrm{D}$ & 1.112 & $\mathrm{NaHCO}_{3}$ & Salobra & Dura \\
\hline & $\mathrm{F}$ & 1.500 & $\mathrm{NaHCO}_{3}$ & Salobra & Dura \\
\hline & 1 & 960 & Mista $\mathrm{HCO}_{3}$ & Salobra & Dura \\
\hline & $J$ & 1.115 & $\mathrm{NaHCO}_{3}$ & Salobra & Dura \\
\hline & $\mathrm{L}$ & 1.168 & Mista $\mathrm{HCO}_{3}$ & Salobra & Dura \\
\hline & $\mathrm{N}$ & 841 & $\mathrm{NaHCO}_{3}$ & Salobra & Dura \\
\hline \multirow{2}{*}{3} & $\mathrm{G}$ & 1.334 & $\mathrm{NaHCO}_{3}$ & Salobra & Dura \\
\hline & $\mathrm{H}$ & 2.321 & $\mathrm{NaCl}$ & Salgada & Dura \\
\hline
\end{tabular}

Fonte: Adaptado de Teramoto et al. (2018) 
No SIG, foram delimitados os três setores (Figura 6), sendo as águas do setor 1 , as de menor salinidade observada, classificadas como doces, de acordo com a Resolução CONAMA (357/2005). Estas águas ocorrem em maior parte sob as condições de água mole para dureza total, de acordo com a classificação de Sawyer et al. (2000). A exceção se dá no ponto A, onde a água apresenta um perfil de água dura, que pode ser explicada em razão de alguma anomalia geológica específica deste ponto, ou influência antrópica, já que, nesta zona, ocorrem algumas atividades industriais.
O setor 2, que tem o maior número de poços analisados, apresenta águas salobras, de acordo com a resolução CONAMA (357/2005). Estas águas são mistas $\mathrm{HCO}_{3}$ ou $\mathrm{NaHCO}_{3}$ e predominantemente duras. Neste setor ocorrem águas com elevados teores de $\mathrm{Cl}^{-}$e forte correlação com o $\mathrm{Na}^{+}$, conforme revela Teramoto et al. (2018), ao indicar que os incrementos destes íons nas águas subterrâneas têm origens distintas. A maior salinidade observada pode estar associada ao intemperismo químico das rochas, deposição atmosférica (aerossóis marinhos) e recarga artificial indireta (águas residuárias).

Figura 6 - Mapa com setores hidroquímicos e proporção de STD para a Zona X

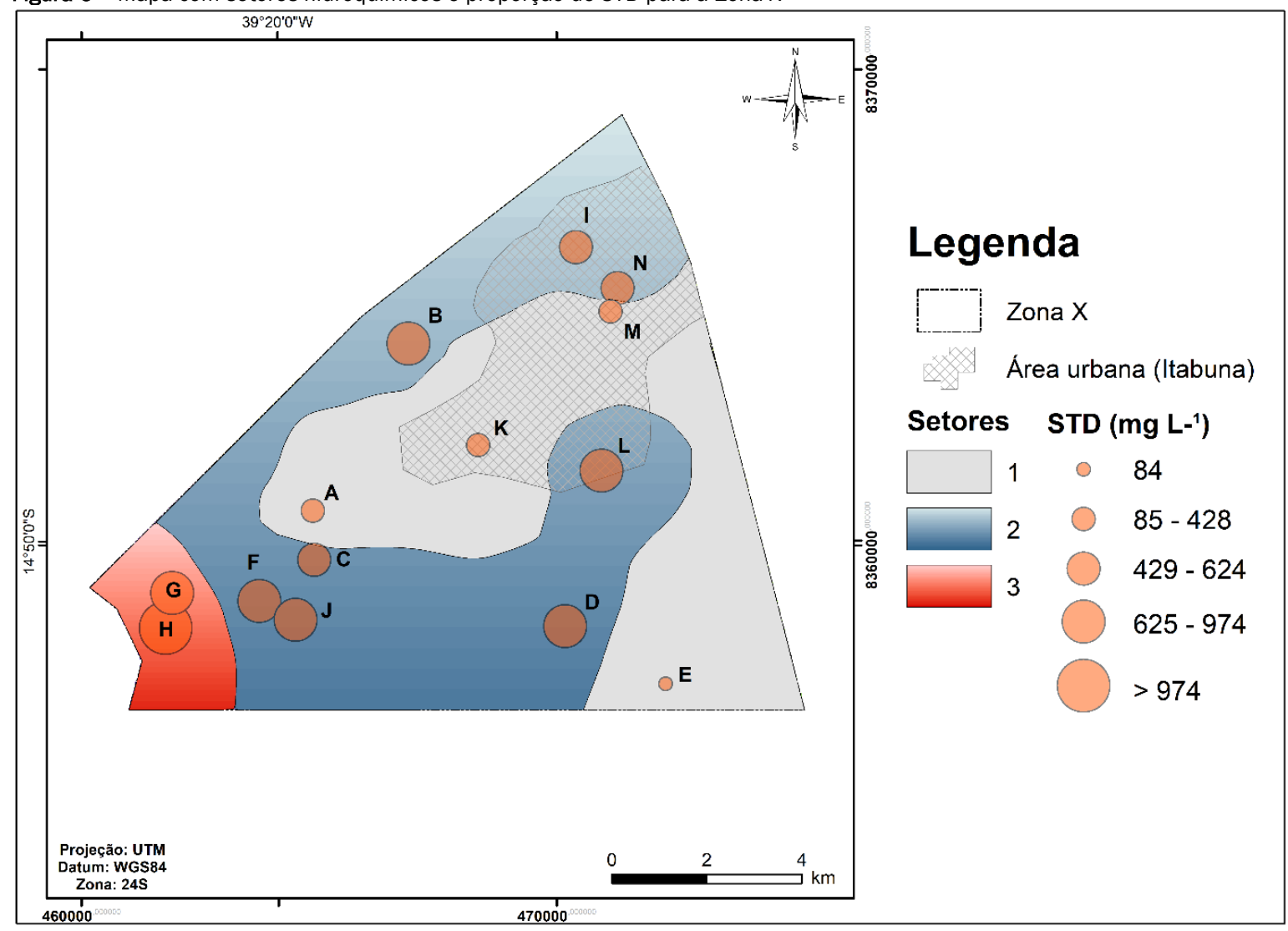

O setor 3, apresenta águas salobras e salgadas, pela Resolução CONAMA 357/2005. São águas duras, dos tipos $\mathrm{NaHCO}_{3}$ e $\mathrm{NaCl}$. O intemperismo químico de rochas como o sienito, é o provável processo que controla a hidroquímica das águas neste setor, conforme mostra De Paula et al. (2009), ao realizar ensaios hidroquímicos em águas superficiais e subterrâneas na região. $\mathrm{A}$ mineralização observada para as águas deste setor, pode ser produto de processos como dissolução mineral, precipitação ou troca iônica. Diop e Tijani (2008), Perrin et al. (2011), apresentam alguns resultados, os quais, mostram que estes processos são comuns no controle da hidrogeoquímica das águas subterrâneas de aquíferos fissurais cristalinos. verão ser executados, sobretudo na escala da área urbana, onde há um recente aumento no número de poços perfurados, além dos problemas de saneamento das águas residuárias que podem implicar negativamente na qualidade das águas subterrâneas. A superexplotação das águas subterrâneas é um problema que pode comprometer o equilíbrio dos aquíferos de uma forma geral, conforme explicam Villar (2016), ao discutir estas águas em um contexto de crise e Molina et al. (2009), ao propor uma análise integrada da gestão hídrica. Com mais dados e análises aprofundadas, será possível compreender o nível de influência das taxas atuais de uso da água subterrânea em Itabuna, além de delimitar com maior precisão os setores hidroquímicos locais.

Vale ressaltar que, estudos mais aprofundados no município de 


\subsection{Contribuições para gestão das águas subterrâneas}

A abordagem apresentada para este estudo, pode efetivamente contribuir com a gestão das águas subterrâneas, a qual, poderá usar as informações geradas pela aplicação do SIG associado ao SOM, para melhor avaliar as decisões relacionadas ao uso da água. Novas metodologias e políticas que promovam a gestão das águas subterrâneas precisam ser encorajadas para buscar soluções de bom custo-benefício para as cidades, além de se adaptarem a cada realidade regional (VASIN et al., 2016; BORGES et al., 2017; SOLDERA \& OLIVEIRA, 2017). No caso de Itabuna, os resultados aqui apresentados podem direcionar um plano diretor que contemple intervenções capazes de dar uma destinação adequada as águas residuárias, tratar as águas impróprias para usos primários, estabelecer algumas normas sanitárias, identificar as zonas prioritárias para proteção das fontes de água, e por consequência mitigar os impactos aos aquíferos.

A demanda por água subterrânea em Itabuna cresceu significativamente, e isso pode ter implicações no aquífero subjacente a área urbana, que apresenta baixo-médio potencial hidrogeológico, reforçando assim a urgente necessidade de formular estratégias para garantir a segurança hídrica de uma cidade média que já enfrenta problemas na oferta e demanda de águas superficiais. Neste contexto, El-Fadel et al. (2014) e Chang et al. (2018), mostram que problemas na produtividade do aquífero e também na qualidade da água precisam ser consideradas por estas estratégias. Como solução, parcerias público-privada podem ser uma alternativa em potencial para otimizar o processo de gestão destas águas. Estas parcerias podem promover o diálogo entre os usuários da água, mitigar e compensar impactos e angariar recursos técnicos, políticos e econômicos para viabilizar as ações necessárias.

O melhor uso e aproveitamento das técnicas empregadas neste estudo, dependem exclusivamente da disponibilidade de dados específicos para hidroquímica, e isso requer investimentos em projetos de monitoramento periódico das águas subterrâneas (EL-ALFY et al., 2015; ABBAS et al., 2018; BELKHIRI et al., 2018). Desta forma, haverá informações para alimentar um banco de dados geográficos a ser usado em um SIG, podendo gerar resultados rápidos, de aplicação prática e uso concreto pela gestão, conforme colocam El-Alfy et al. (2015) e Aydi (2018).

\section{CONCLUSÕES}

As técnicas empregadas para o zoneamento hidroquímico das águas subterrâneas na cidade de Itabuna mostram que, se as características hidroquímicas forem conhecidas para uma determinada unidade espacial, é possível delimitar setores com o uso do SIG e do SOM. As análises espaciais no SIG geraram um padrão, e o SOM com seu caráter multivariado, refinou este padrão e definiu o traçado para o zoneamento.

Com as similaridades e diferenças identificadas entre as estações amostrais, foi possível dividir as águas em três setores. Águas com menor salinidade, doces e predominantemente mo- les ocorrem na área dos metatonalitos e em boa parte do território urbano (setor 1). Águas salobras e duras ocorrem na faixa das rochas granuliticas no setor 2. Águas de maior salinidade, salgadas e duras ocorrem na faixa oeste, no setor 3 , com provável influência das rochas alcalinas (sienitos). Vale ressaltar que os resultados não refletem necessariamente uma estimativa conclusiva destes setores, mas, conforme discutido, apresentam coerência diante das evidências encontradas.

As análises espaciais executadas ajudam a elucidar os padrões hidroquímicos de cada setor, e os mapas (cartográfico e autoorganizáveis) gerados propõem inúmeras alternativas de aplicação para gestão de recursos hídricos em qualquer cidade no mundo. Com o subsidio do SIG e do SOM, problemas de uso da água em cidades podem ser solucionados a partir de uma perspectiva espacial-estatística que poderá promover uma efetiva interação da gestão urbana com a segurança hídrica, bem como, direcionar políticas de controle da qualidade das águas subterrâneas, coordenar ações de proteção dos aquíferos e executar intervenções de engenharia que busquem sanear as águas deterioradas para usos diversos.

\section{AGRADECIMENTOS}

Agradecemos ao Dr. Elias H. Teramoto e ao Laboratório de Estudos de Bacias da UNESP, pela fundamental colaboração no fornecimento dos dados para este estudo. A CAPES (código 001) e FAPESP (Projeto número 18/09401-1) pelo suporte técnico e financeiro.

\section{REFERÊNCIAS}

ABBAS, Z.; MAPOMA, H. W. T.; SU, C.; AZIZ, S. Z.; MA, Y.; ABBAS, N. Spatial analysis of groundwater suitability for drinking and irrigation in Lahore, Pakistan. Environmental Monitoring and Assessment, v. 190, n. 391, 2018.

ALVARES, C. A.; STAPE, J. L.; SENTELHAS, P. C.; GONÇALVES, J. L. M.; SPAROVEK, G. Köppen's climate classification map for Brazil. Meteorologische Zeitschrif, v. 22, n. 6, p. 711-728, 2013.

AYDI, A. Evaluation of groundwater vulnerability to pollution using a GIS-based multi-criteria decision analysis. Groundwater for Sustainable Development, v. 7, p. 204-211, 2018.

BARROS, J. F.; ALMEIDA, J. B.; SILVA, C. M. S. V.; MENDES FILHO, J.; MESQUITA, B. A.; SANTIAGO, M. M. F.; FRISCHKORN, H. Caracterização isotópica $(18 \mathrm{O}, \mathrm{D})$ e hidroquímica das águas da bacia do rio da Prata de São Luís/MA e vizinhança. Revista Brasileira de Recursos Hídricos, v. 20, n. 2, p. 404-410, 2015.

BELKHIRI, L.; MOUNI, L.; TIRI, A.; NARANY, T. S.; NOUIBET, R. Spatial analysis of groundwater quality using self-organizing maps. Groundwater for Sustainable Development, v. 7, p. 121 132, 2018. 
BORGES, V. M.; ATHAYDE, G. B.; REGINATO, P. A. R. Avaliação da vulnerabilidade natural à contaminação do sistema aquífero Serra Geral no Estado do Paraná - Brasil. Águas Subterrâneas, v. 31, n. 4, p. 327-337, 2017.

BRASIL. Conselho Nacional do Meio Ambiente - CONAMA. Resolução 357, de 17 de março de 2005. Dispõe sobre a classificação dos corpos de água e diretrizes ambientais para o seu enquadramento, bem como estabelece as condições e padrões de lançamento de efluentes, e dá outras providências. BrasíliaDF, 2005.

CHANG, F-J.; HUANG, C-W.; CHENG, S-T.; CHANG, L-C. Conservation of groundwater from over-exploitation-Scientific analyses for groundwater resources management. Science of The Total Environment, v. 598, p. 828-838, 2017.

DE PAULA, F. C. F.; SILVA, D. M. L.; SOUZA, C. M. Tipologias hidroquímicas das bacias hidrográficas do Leste da Bahia. Revista Virtual de Química, v. 4, n. 4, p. 365-373, 2009.

DIOP, S.; TIJANI, M. N. Assessing the basement aquifers of Eastern Senegal. Hydrogeology Journal, v. 16, n. 7, p. 1349-1369, 2008.

EL ALFY, M.; LASHIN, A.; AL-ARIFI, N.; AL-BASSAM, A. Groundwater characteristics and pollution assessment using integrated hydrochemical investigations GIS and multivariate geostatistical techniques in arid áreas. Water Resources Management, v. 29, n. 15, p. 5593-5612, 2015.

EL-FADEL, M.; TOMASZKIEWICZ, M.; ADRA, Y. et al. GIS-Based Assessment for the Development of a Groundwater Quality Index Towards Sustainable Aquifer Management. Water Resources Management, v. 28, n. 11, p. 3471-3487, 2014.

FRIEDEL, M. J.; SOUZA-FILHO, O. A.; IWASHITA, F.; SILVA, A. M.; YOSHINAGA, S. Data-driven modeling for groundwater exploration in fractured crystalline terrain, northeast Brazil. Hydrogeology Journal, v. 20, n. 6, p. 1061-1080, 2012.

GLEESON, T.; BEFUS, K. M.; JASECHKO, S.; LUIJENDIJK, E.; CARDENAS, M. B. The global volume and distribution of modern groundwater. Nature Geoscience, v. 9, p. 161-167, 2016.

GOMES, M.C.R.; CAVALCANTE, I. N. Análise geoquímica das águas subterrâneas de Fortaleza, Ceará - Brasil. Águas Subterrâneas, v. 29, n.1, p. 42-59, 2015.

GOMES, M. A.; RAMOS, E. V. S.; SANTOS, L. C.; BITU, S. G.; GADELHA, A. J. F. Avaliação hidroquímica e de parâmetros físicoquímicos de qualidade das águas subterrâneas da zona urbana do município de Sousa-PB. Águas Subterrâneas, v. 32, n. 2, p. 162-172, 2018.

GUDE, V. J. Desalination of deep groundwater aquifers for fresh water supplies - Challenges and strategies. Groundwater for
Sustainable Development, v. 6, p. 87-92, 2018.

HASSANE, A. B.; LEDUC, C.; FAVREAU, G.; BEKINS, B. A.; MARGUERON, T. Impacts of a large Sahelian city on groundwater hy drodynamics and quality: example of Niamey (Niger). Hydrogeology Journal, v. 24, n. 2, p. 407-423, 2016.

IBGE. Instituto Brasileiro de Geografia e Estatística. Cidades Estimativas de população (2017). Disponível em: <http://www.ibge.gov.br/home/estatistica/populacao/estimativa2016/estimativa_tcu.shtm>. Acesso em: 22 abril, 2018.

KOHONEN, T. Self-organized formation of topologically correct feature maps. Biological Cybernets, v. 43, n. 1, p. 59-69, 1982.

KOHONEN, T. Essentials of the self-organizing map. Neural Networks, v. 37, p. 52-65, 2013.

KOPPEN, W. Climatologia con un estudio de los climas de la tierra (transl. P. R. H. Peres), Fondo de Cultura e Economica, Mexico City, Mexico, 1948.

MACHIWAL, D.; JHA, M. K. Identifying sources of groundwater contamination in a hard-rock aquifer system using multivariate statistical analyses and GIS-based geostatistical modeling techniques. Journal of Hydrology: Regional Studies, v. 4, n. A, p. 80110, 2015.

MAIA, P. H. P.; CRUZ, M. J. M.; SAMPAIO, M. C. Zoneamento dos aquíferos do estado da Bahia. Brazilian Journal of Aquatic Science and Technology, v. 13, n. 1, p. 45-52, 2009.

MARGAT, J.; VAN DER GUN, J. Groundwater Around the World: A Geographic Synopsis, 376. Leiden, The Netherlands, 2013.

MATTOS, J. B.; SILVA, K. B.; SILVA, R. J.; ALMEIDA, T. H. M.; POVOAS, H. S. S.; SILVA, P. V. R., GÓES, I. M. A.; MATOS, I. S. Natural factors or environmental neglect? Undestanding the dilemma of a water crisis in a scenario of water plenty. Land Use Policy, v. 82, p. 509-517, 2019.

MELO, J. G.; VASCONCELOS, M. B.; ALVES, R. S. Origem e mecanismos de salinização das águas subterrâneas na borda sul da bacia Potiguar, RN. Revista Brasileira de Recursos Hídricos, $v$. 17, n. 4, p. 161-169, 2012.

MENEZES, J. P. C.; BERTOSSI, A. P. A.; SANTOS, A. R.; NEVES, M. A. Correlação entre uso da terra e qualidade da água subterrânea. Engenharia Sanitária e ambiental, v.19, n. 2, p. 173-186, 2014.

MOLINA, J. L.; ARÓSTEGUI, J. L. C.; BENAVENTE, J. et al. Aquifers Overexploitation in SE Spain: A Proposal for the Integrated Analysis of Water Management. Water Resources Management, v. 23, n. 13, p. 2737-2760, 2009. 
PERRIN, J.; AHMED, S.; HUNKELER, D. The effects of geological heterogeneities and piezometric fluctuations on groundwater flow and chemistry in a hard-rock aquifer, southern India. $\mathrm{Hy}$ drogeology Journal, v. 19, p. 1189-1201, 2011.

QIAN, J.; WANG, L.; ZHAN, H.; CHEN, Z. Urban land-use effects on groundwater phosphate distribution in a shallow aquifer, Nanfei River basin, China. Hydrogeology Journal, v. 19, n. 7, p. 1431-1442, 2011.

SAWYER, C. N.; McCARTY, P. L.; PARKIN, G. F. Chemistry for sanitary engineers. 4. ed. New York, McGraw-Hill, 2000.

SEGEV, A.; KANTOLA, J. Identification of trends from patents using self-organizing maps. Expert Systems with Applications, v. 39 , n. 18, p. 13235-13242, 2012.

SHULER, C. K.; EL-KADI, A. L.; DULAI, H.; GLENN, C. R.; FACKRELL, J. Source partitioning of anthropogenic groundwater nitrogen in a mixed-use landscape, Tutuila, American Samoa. Hydrogeology Journal, v. 25, n. 8, p. 2419-2434, 2017.
SOLDERA, B. C.; OLIVEIRA, E. Água sustentável (AS): um novo método para a governança da água. Águas Subterrâneas, v. 31, n. 2, p. 30-43, 2017.

TERAMOTO, E. H.; ENGELBRECTH, B. Z.; GONÇALVES, R. D.; CHANG, H. K. Caracterização hidroquímica e isotópica dos aquíferos fissurais da região de Itabuna/BA. Águas Subterrâneas, v. 32, n. 2, p. 228-236, 2018.

THE MATHWORKS INCORPORATED. MatLab R2018a. Natick, MA, United States, 2018.

VASIN, S.; CARLE, A.; LANG, U.; KIRCHHOLTES, H. J. A groundwater management plan for Stuttgart. Science of The Total Environment, v. 563-564, p. 704-712, 2016.

VILLAR, P. C. As águas subterrâneas e o direito à água em um contexto de crise. Ambiente \& Sociedade, v. 19, n. 1, p. 85102, 2016. 